\title{
Acute Onset of Chronic Inflammatory Demyelinating Polyneuropathy: A Case Report
}

\author{
Jie Wu ${ }^{\mathrm{a}}$, Chunling Song ${ }^{\mathrm{a}}$, Yanqiu Han ${ }^{\mathrm{a}}$, Shanji Nan ${ }^{\mathrm{a}} \mathrm{b}$
}

\begin{abstract}
Acute onset chronic inflammatory demyelinating polyneuropathy (ACIDP) with facial weakness and mild respiratory muscle weakness in the three episodes is rarely reported. We describe a Chinese man with A-CIDP who experienced weakness in both upper limbs as well as facial muscles, and his condition deteriorated in three different consultations over the span of 14 weeks. Patients with A-CIDP were more likely to have prominent sensory signs rather than motor signs and were less likely to have autonomic dysfunction, facial weakness, or need for mechanical ventilation. Our patient had facial weakness in the first and second episodes, and mild respiratory muscle weakness in the first and third episodes; hence, our patient was most likely an A-CIDP case different from those cases in the literature. Early recognition of A-CIDP in patients with apparent GBS is important so that proper diagnostic and therapeutic management can be initiated promptly.
\end{abstract}

Keywords: Chronic inflammatory demyelinating polyneuropathy; Guillain-Barre syndrome

\section{Introduction}

Chronic inflammatory demyelinating polyneuropathy (CIDP) is an acquired immune-mediated inflammatory disorder of the peripheral nervous system which has clinical similarities to acute inflammatory demyelinating disease of the peripheral nerves called Guillain-Barre syndrome (GBS) [1, 2]. Sixteen percent of patients with acute CIDP (A-CIDP) experience rapid deterioration within 8 weeks followed by a chronic course, while $6-16 \%$ of treated patients with GBS have one or more deteriorations after initial improvement that is typically described as treatment-related fluctuations (TRFs) [3, 4]. Here we report a case of a 49-year-old male patient with A-CIDP

Manuscript accepted for publication June 08, 2016

aDepartment of Neurology, the Second Hospital of Jilin University, Changchun 130041, China

${ }^{b}$ Corresponding Author: Shanji Nan, Department of Neurology, the Second Hospital of Jilin University, Changchun 130041, China.

Email: xuchengbi@hotmail.com

doi: https://doi.org/10.14740/jmc2550w presenting with apparent GBS.

\section{Case Report}

A 49-year-old male was admitted to the hospital on November 1, 2012 for syndrome of weakness in the facial muscles and progressive loss of sensation that began in his upper limbs and progressed distally. One day prior to admission, the patient suffered weakness in his legs, and walked slowly with an unsteady gait. He also presented with slurred speech and dysphagia. According to the patient, he had intermittent diarrhea for a month before the onset of the syndrome.

Physical examination findings and electromyogram (EMG) characteristics are summarized in Table 1. The patient developed mild dyspnea on his second day of admission. On day 3, a lumbar puncture was performed and the results showed increased protein content (Table 1).

The patient was initially diagnosed with GBS, stage II hypertension (moderate to severe), and viral hepatitis. Owing to financial constraints, the patient could not afford gamma globulin replacement therapy or plasma exchange. He opted for steroid treatment instead. He took methylprednisolone (60 $\mathrm{mg}$ orally once daily) for 5 days followed by tapering of the dose by $5 \mathrm{mg}$ every 2 days. The patient was given an intramuscular injection of vitamin B1, B12 and mouse nerve growth factor. His symptoms improved progressively, and the lower limb muscle strength recovered to the point where he could carry heavy loads with a normal gait. The patient's speech returned to normal, and his dysphagia and dyspnea subsided. The sensation in all his limbs was normal. He had intermittent sensation loss on his back. He was discharged after 8 days, while still taking a daily dose of $50 \mathrm{mg}$ methylprednisolone, $100 \mathrm{mg}$ vitamin B1, and $500 \mu \mathrm{g}$ methylcobalamin. The dose of methylprednisolone was gradually reduced until cessation by $5 \mathrm{mg}$ every 2 days.

Fourteen weeks after the first episode, the patient drank 10 $\mathrm{mL}$ of alcohol. He experienced slurred speech, weakness in his lower limbs, and inability to walk. He was readmitted to the hospital on February 1, 2013.

Physical examination findings and EMG characteristics are summarized in Table 1. On day 5, the patient underwent lumbar puncture. The results showed increased protein content (Table 1). Examination for anti-ganglioside antibody spectrum 2 (GM1, GM2, GM3, GD1a, GD1b, GT1b, and GQ1b) was negative (Euroimmun). Based on his clinical features and disease progression, the patient was differentially diagnosed 


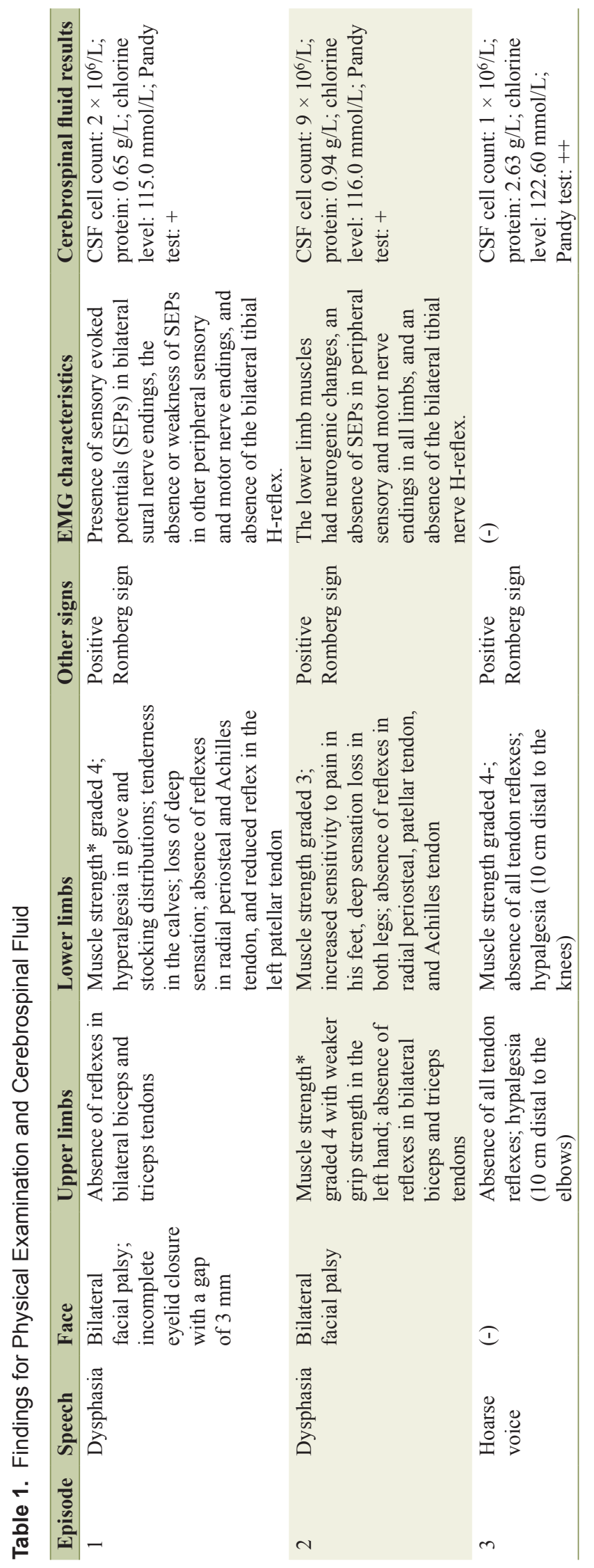

with A-CIDP. He restarted taking $60 \mathrm{mg}$ methylprednisolone daily for 5 days followed by weaning of the dose of methylprednisolone by $5 \mathrm{mg}$ per week. He received an intramuscular injection of vitamin B1, B12 and mouse nerve growth factor. Three weeks after this admission, the patient's symptoms had improved. His speech was nearly normal. Bilateral peripheral facial palsy had partially recovered without leaking of his breath when he blew his cheeks. He could walk slowly. The patient was discharged from the hospital while still taking methylprednisolone which was gradually tapered until cessation by $5 \mathrm{mg}$ per week.

Eight weeks after the second episode, the patient manifested recurrent symptoms for the third time. He suffered limb weakness and slurred speech. He was able to stand but could not walk, and he had shortness of breath. Two weeks after the recurrent symptoms, he was readmitted to the hospital. Findings for physical examination and lumbar puncture are summarized in Table 1. The patient was given pulse methylprednisolone treatment. Two weeks after the pulse treatment, the clinical manifestations had improved. His speech returned to normal. He had atrophy in the first dorsal interosseous muscle in both hands. His upper-limb muscle strength was graded 5-, and lower-limb muscle strength was graded $4+$. The symptom of hypalgesia had not improved. The patient was discharged 2 weeks after the treatment and he was followed up as an outpatient. One year after his discharge, the patient's status was updated by a follow-up phone call. According to his description, he was able to perform daily chores and routines, his strength was slightly weaker than normal, and no other abnormality was reported.

\section{Discussion}

Ruts et al [5] suggested A-CIDP should be suspected when a patient with GBS deteriorates after 9 weeks from the onset or when deterioration occurs three or more times. In this case, the patient's condition deteriorated over 14 weeks after the first onset and the deterioration occurred three times; hence, he was diagnosed with A-CIDP according to the key diagnostic features described by Ruts et al [5]. In addition, Dionne et al performed a retrospective review on 30 patients with acute inflammatory demyelinating polyneuropathy (AIDP) and 15 patients with A-CIDP and revealed that infectious prodrome was rarely correlated with A-CIDP; patients with A-CIDP were more likely to have prominent sensory signs rather than motor signs. In their studies, 53.3\% A-CIDP patients presented with signs of sensory ataxia, impaired vibration perception, and stocking-glove hypalgesia, which was consistent with the symptoms experienced by our patient. Nevertheless, Dionne et al also reported that patients with A-CIDP were less likely to have autonomic dysfunction, facial weakness, or need for mechanical ventilation [6]. Ruts et al performed a prospective longitudinal study on patients with GBS-TRF and with A-CIDP in order to distinguish these two diseases in the early phase of disease. Their results revealed, at all time points, ACIDP group was less severely affected than GBS-TRF group in level of weakness and severity. A-CIDP patients did not 
need artificial ventilation, had significantly less cranial nerve dysfunction, and tended to have more CIDP-like electrophysiologic abnormalities. In Rut's study, only one out of eight A-CIDP patients appeared cranial nerve dysfunction (VII). However, in this case, our patient had facial weakness in the first and second episodes, and mild respiratory muscle weakness in the first and third episodes; hence, our patient was an A-CIDP case different from those cases reported in the previous literatures.

Early recognition of A-CIDP in patients with apparent GBS is clinically difficult, but important because it can lead clinicians to initiate steroid therapy. While A-CIDP requires maintenance therapy after the initial response to steroid treatment, adjuvant immunosuppressive therapy is commonly used to lower the steroid dose, thereby reducing side effects [4].

\section{Acknowledgments}

The authors would like to thank Medjaden Bioscience Limited for providing assistance in the preparation of this manuscript.

\section{Conflicts of Interest}

No potential conflicts of interest relevant to this article were reported by all authors of the study.

\section{References}

1. Dyck PJ, Lais AC, Ohta M, Bastron JA, Okazaki H, Groover RV. Chronic inflammatory polyradiculoneuropathy. Mayo Clin Proc. 1975;50(11):621-637.

2. Koller H, Kieseier BC, Jander S, Hartung HP. Chronic inflammatory demyelinating polyneuropathy. N Engl J Med. 2005;352(13):1343-1356.

3. Robertson EE, Donofrio PD. Treatment of chronic inflammatory demyelinating polyneuropathy. Curr Treat Options Neurol. 2010;12(2):84-94.

4. Hadden RD. Deterioration after Guillain-Barre syndrome: recurrence, treatment-related fluctuation or CIDP? J Neurol Neurosurg Psychiatry. 2009;80(1):3.

5. Ruts L, van Koningsveld R, van Doorn PA. Distinguishing acute-onset CIDP from Guillain-Barre syndrome with treatment related fluctuations. Neurology. 2005;65(1):138-140.

6. Dionne A, Nicolle MW, Hahn AF. Clinical and electrophysiological parameters distinguishing acute-onset chronic inflammatory demyelinating polyneuropathy from acute inflammatory demyelinating polyneuropathy. Muscle Nerve. 2010;41(2):202-207. 